\title{
OTIOTOMICS
}

Revista de economía, empresa y sociedad

\section{Realidad aumentada y turismo. Potenciales y límites para la mejora de la competitividad en los destinos turísticos}

\author{
Francesc González Reverté \\ Profesor de la Universitat Oberta de Catalunya (UOC). Estudios de Economía y Empresa
}

RESUMEN Este artículo pretende ser una aproximación al potencial que la realidad aumentada genera en el sector turístico, uno de los sectores económicos que menos la ha usado y en el que mayores expectativas se han puesto. La realidad aumentada es una tecnología disruptiva que, bien gestionada, puede resultar un importante instrumento de competitividad para los destinos turísticos. En especial, la combinación de la realidad aumentada con los dispositivos móviles, hoy en día de uso prácticamente universal entre los turistas, genera altas expectativas en relación con la captación de aquellos perfiles de turistas más tecnológicos, como los llamados millenials.

La realidad aumentada mejora la experiencia turística de los visitantes y permite la creación de nuevos productos, así como formas creativas de promocionar los destinos a bajo coste. No obstante, antes de implementar acciones estratégicas centradas en el uso de la realidad aumentada, es conveniente conocer qué limitaciones tiene en términos de usabilidad tecnológica, desconocimiento de las preferencias de la demanda y falta de evaluación de los resultados y del rendimiento económico obtenido.

PALABRAS CLAVE realidad aumentada; dispositivos móviles; turismo; nuevas tecnologías; competitividad; millenials

Augmented Reality and Tourism. Potential and limits for the improvement of competitiveness in tourism destinations

ABSTRACT This paper intends to be an approach to the potential of augmented reality in the tourism sector, one of the economic sectors in which expectations are higher but where it has been scarcely applied. Augmented reality is a disruptive technology that, if properly managed, can be an important tool for tourist destinations in terms of competitiveness. In particular, the combination of augmented reality with mobile devices generates high expectations in relation to the attraction of technological tourists, best known as millennials. Augmented reality enhances the tourist experience of the visitors and enables the creation of new tourism products and low cost creative ways to 
promote destinations. However, before carrying out strategic actions, the tourist managers should be conscious of the limits of augmented reality in terms of technological usability, preferences lack of demand and lack of evaluation of results and economic performance obtained.

KEYWORDS augmented reality; mobile devices; tourism; new technologies; competitiveness; millennials

\section{Introducción}

Este artículo pretende ofrecer una visión panorámica del potencial de las tecnologías de realidad aumentada como instrumento para la mejora de la competitividad de los destinos turísticos. En primer lugar, se ofrece una introducción a la importancia que el uso de internet y los dispositivos móviles está teniendo actualmente en la transformación de la experiencia turística. Asimismo, se explican cuáles son las características y factores de la realidad aumentada que la convierten en una herramienta con enorme potencial para proporcionar valor añadido a la visita turística mediante el uso de dispositivos móviles. En este sentido, se exponen las opciones y las potencialidades que la realidad aumentada ofrece para generar nuevas experiencias sensoriales durante la visita turística. Finalmente, se hace mención de los principales problemas y limitaciones que plantea el uso de la realidad aumentada al servicio del turismo desde dos puntos de vista: la satisfacción del usuario y la capacidad que los proyectos basados en la realidad aumentada tienen para generar procesos reales y continuados de desarrollo turístico sobre el territorio.

\section{Los dispositivos móviles y la realidad aumentada como referente de las nuevas tecnologías en turismo}

\subsection{Las nuevas tecnologías en turismo}

La tecnología siempre ha sido un pilar de innovación para el turismo. Desde la aparición de internet la alianza con la tecnología ha supuesto grandes transformaciones en los procesos de producción y consumo turístico que se han manifestado en varias evidencias. Algunas de las más importantes han sido la reducción de costes del producto, el nuevo enfoque de los procesos de intermediación con afectación sobre los precios finales de venta, la contribución a la expansión global del turismo (y al incremento exponencial de las opciones de elegir un lugar para disfrutar de las vacaciones) o la creación de nuevas relaciones entre los usuarios (los que tienen una mayor participación activa) y los proveedores. Se considera que internet ha sido una de las tecnologías que más radicalmente ha transformado la actitud de los consumidores y el comportamiento de los turistas (Buhalis y Law, 2008; Buhalis y O'Connor, 2005). Entre los cambios más destacados encontramos la posibilidad de los consumidores de interactuar directamente con los proveedores (y, por tanto, modificando el rol de los intermediarios) (Jeong, Oh y Gregoire, 2003) y con los destinos (y, por tanto, pedir directamente productos y servicios más personalizados y de acuerdo con sus necesidades). La rápida transmisión de datos que genera el uso de internet también cambia el tiempo de respuesta esperado por los consumidores, el cual se reduce considerablemente y obliga a las organizaciones a gestionar la información más eficientemente. Por otra parte, las reacciones a las demandas hechas en línea por los consumidores pueden afectar considerablemente a la satisfacción y la decisión de hacer una reserva, por lo que la respuesta a las peticiones y solicitudes de los clientes es un factor esencial para el éxito de las pequeñas y medianas empresas turísticas, tanto desde el punto de vista de la reputación como de la lealtad (Main, 2001). 
Si bien en los primeros años de la llegada de internet los ordenadores personales eran el instrumento de gestión en línea predominante, en los últimos años los dispositivos móviles están tomando el protagonismo. Las consultas de información y las reservas a través de los dispositivos móviles son cada vez más habituales entre la mayor parte de los usuarios. Los expertos señalan que la razón principal del éxito y expansión de los dispositivos móviles en turismo es la facilidad y rapidez con que permiten obtener soluciones e información en tiempo real que cubren las necesidades de los turistas de manera satisfactoria. Algunos autores afirman que el uso de los dispositivos móviles forma parte de la propia experiencia turística de los usuarios, ya que, como sucede con la realidad aumentada, permiten amplificar los aspectos sensoriales de la visita (Lamfus y otros, 2013).

Un segundo elemento importante que hay que tener presente es que el aumento de la información disponible de forma inmediata a través de los dispositivos móviles puede alterar el contexto de toma de decisiones, especialmente cuando se está en movimiento o en el destino, ya que los dispositivos móviles son un instrumento ideal para decidir sobre diferentes opciones de manera rápida, inmediata, espontánea y con ahorro de tiempo. Por ejemplo, el uso de aplicaciones que recogen recomendaciones de otros usuarios (como TripAdvisor) o de los propios amigos (como FourSquare) basados en la propia experiencia pueden hacer tomar nuevas decisiones espontáneas a los turistas que visitan un destino.

\subsection{La realidad aumentada y la experiencia turística}

En este sentido, la realidad aumentada es una de las tecnologías que mejor puede contribuir a mejorar la experiencia turística en el lugar de destino. Por realidad aumentada se entiende un entorno que incluye, al mismo tiempo, elementos de realidad virtual y del mundo real, los cuales pueden interactuar entre sí. Es decir, a diferencia de la realidad virtual, donde todo es ficticio, un sistema de realidad aumentada mejora o enriquece el entorno que está siendo observado con información virtual que parece que coexista con el mundo real. Cuando la realidad aumentada se combina con dispositivos móviles permite su uso en el exterior simplemente apuntando el aparato hacia un objeto físico y sus alrededores. Entonces es posible obtener información adicional sobre este objeto (vídeo, audio, imágenes, texto, símbolos, marcadores, etc.) superpuestos en el elemento del mundo real que se tiene en pantalla mediante anotaciones de información virtual (González y otros, 2012).

¿Por qué la realidad aumentada está teniendo tanto éxito? Desde el punto de vista de los turistas, básicamente estamos ante una tecnología de bajo coste que, mediante un smartphone o una tableta, puede ser accesible a casi todo tipo de usuarios y porque aumenta de forma exponencial las posibilidades de interactuar y obtener información del entorno. Desde el punto de vista de los negocios turísticos, porque la realidad aumentada en general implica un efecto sorpresa, el cual genera la difusión de boca en boca, y porque favorece la innovación y el posicionamiento ante los competidores. Desde el punto de vista de los destinos, además de todos los factores antes mencionados, hay que tener en cuenta el hecho de que la realidad aumentada permite ampliar la visita turística real y crear sinergias con los visitantes. A medida que los destinos sean inteligentes e introduzcan el big data en su gestión, la experiencia de visitar ciudades se nutrirá de información creada por los destinos (servicios de atención al cliente, redes comerciales y de entidades culturales, servicios de información, etc.) pero también por los propios usuarios. Además, será posible conocer mucho mejor y en tiempo real las preferencias y gustos de los visitantes sobre los servicios, productos y lugares turísticos visitados.

\section{Potencialidades y límites de la realidad aumentada para la mejora de la competitividad de los destinos turísticos}

La realidad aumentada tiene un largo recorrido en diversos ámbitos de la vida cotidiana, pero en turismo está infrautilizada y aún queda mucho por hacer, especialmente como elemento de negocio. Los usos más conocidos de realidad aumentada en turismo se centran en el mundo del patrimonio y los museos; por ejemplo, el museo de Canterbury utiliza prismáticos digitales para dar vida a los elementos estáticos expuestos y el London Museum 
hace uso de la app Streetmuseum para hacer itinerarios visualizando in situ imágenes históricas de los lugares visitados. Pero hay muchas más posibles aplicaciones ${ }^{1}$, como el uso de 3D para diseñar catálogos turísticos animados, la visualización de escenarios urbanos en 3D tal como eran y como pueden ser en el futuro, las mesas interactivas digitales de restaurantes, donde se pueden hacer los pedidos y obtener información de los platos, o en educación y formación (como hace la UOC, en su grado de Turismo, usando la realidad aumentada como medio para dar valor añadido y mejorar la interpretación de un recurso turístico esencial como es el paisaje). Como toda nueva tecnología, antes de implantarla es conveniente reflexionar sobre las potencialidades y los límites que plantea su uso. Los beneficios incluyen tanto a los turistas como a la industria. Los turistas, en su mayoría usuarios de dispositivos móviles, aumentan la interacción con el destino y obtienen información en tiempo real que les permite tener nuevas perspectivas de interpretación y comprensión de lo que visitan. Para el sector, la realidad aumentada puede funcionar como una especie de marketing de bajo coste que mejora el compromiso del turista con la marca añadiendo valor a su experiencia. Los límites tienen que ver con la usabilidad y el diseño (hay que tener en cuenta que algunos usuarios simplemente se cansan de llevar en la mano un aparato o que les molestan los reflejos del sol en la pantalla), con la capacidad de personalizar contenidos de realidad aumentada que se presten a las necesidades de cada individuo (hay estudios que afirman que las preferencias entre hombres y mujeres en el uso de tecnologías de realidad aumentada son considerables) o con evitar un exceso de información que sature al usuario.

\subsection{Potencialidades de la realidad aumentada aplicada al turismo}

Los destinos turísticos, para seguir siendo competitivos en los mercados globales, requieren una constante inversión en nuevas tecnologías y, en especial, estar atentos a las innovaciones en los dispositivos móviles. En el contexto turístico actual, donde todavía el uso de la realidad aumentada es incipiente, la combinación de esta con los dispositivos móviles puede proporcionar considerables ventajas competitivas a los destinos que introduzcan proyectos pioneros. Destacamos dos tipos de potenciales impactos positivos de la realidad aumentada. En primer lugar, se trata de tecnologías gratuitas y de fácil uso que se corresponden con los nuevos perfiles de turistas, como los millenials, siempre conectados y que acompañan de prestaciones tecnológicas a todas las fases de su estancia turística. Se trata de viajeros sofisticados y experimentados y muy sensibles a la calidad del servicio y a la personalización de la visita. La realidad aumentada permite mejorar las prestaciones para estos tipos de consumidor turístico aportando información personalizada, aumentando la interacción con los proveedores y reduciendo el tiempo de respuesta en la búsqueda de información. Es decir, desde el punto de vista de la atracción de nueva demanda y de la comercialización de los destinos, la introducción de la realidad aumentada como elemento de la visita turística puede permitir segmentar mejor los mercados objetivo y mejorar su satisfacción con la visita.

El segundo beneficio potencial deriva de la capacidad que la realidad aumentada tiene para mejorar la experiencia turística del visitante. La realidad aumentada ofrece información en tiempo real sobre los objetos de interés, a los que sitúa en un contexto reconocible. Esta inmediatez representa una ayuda significativa por los turistas, la gran mayoría de los cuales visitan un lugar de cuyo entorno desconocen casi todo (Yovcheva y otros, 2012). Además, desde el punto de vista del usuario, la experiencia turística puede verse radicalmente transformada mediante la realidad aumentada (Agüero y González, 2014). La capacidad de interactuar mejor, de personalizar y de entretener que aporta la realidad aumentada son aspectos que se deben considerar para obtener experiencias memorables. Por ejemplo, los turistas que exploran el destino o visitan un museo pueden añadir capas de información de su interés a la realidad que ven, prescindiendo de aquella información que no les interesa. Por otro lado, la realidad aumentada aporta mejoras en la visita. Por ejemplo, haciendo de sustituto de la información de señalización (paneles, etc.) que estorba o dificulta la visualización en lugares de patrimonio sensibles, innovando en la interpretación de los objetos expuestos en museos (por ejemplo, incorporando criterios de edutainment), visualizando los elementos patrimoniales desde nuevos puntos

1. Para más información sobre los usos de la realidad aumentada en turismo pueden ver las intervenciones de los diferentes ponentes de la IV Jornada Know Tour (2015), organizada por el Grupo de Investigación Laboratorio del Nuevo Turismo de la UOC sobre realidad aumentada y turismo. 
de vista, creando viajes en el tiempo que permiten recuperar la memoria histórica, o facilitando la visita a personas con deficiencias sensoriales (Jung y Han, 2014). Otros autores añaden que las diferentes prestaciones y escenarios específicos de la realidad aumentada (visión de objetos en 3D, reconstrucciones, personajes que cobran vida, etc.) permiten mejorar diferentes factores de la experiencia turística (atracción, motivación, compromiso, novedad y seguridad) (Fritz y otros, 2005; Yovcheva y otros, 2013).

\subsection{Límites en el uso de la realidad aumentada en turismo}

Las enormes potencialidades que incluye el uso turístico de la realidad aumentada no deben impedir visualizar los riesgos que van asociados al uso incipiente de esta tecnología. Encontramos cuatro tipos de limitaciones y riesgos. El primer riesgo se relaciona con la capacidad de durar en el tiempo que la propia tecnología puede tener. Todas las tecnologías tienen un ciclo de vida, ya sea más largo o más corto, lo que puede dificultar la construcción de estrategias a largo plazo. El abaratamiento de los costes y la aparición de nuevos gadgets y aparatos que integran la realidad aumentada (las gafas Google Glass y los wearables son algunos de los ejemplos más conocidos), así como la tendencia a incorporar novedades (la visión en $360^{\circ}$, el uso del vídeo, hologramas, etc.), parecen garantizar la expansión de esta tecnología entre los consumidores, pero el riesgo de sustitución tecnológica siempre se debe tener presente. La velocidad del cambio tecnológico también representa una limitación cuando se contrasta con la dinámica temporal de la planificación turística, de formulación mucho más lenta.

Una segunda limitación tiene que ver con las características de usabilidad de la propia tecnología, sobre todo teniendo en cuenta que se aplica a partir de los dispositivos móviles. Aspectos prácticos como la duración de la batería, el peso, la reflexión del sol en la pantalla, el coste del roaming o la falta de zonas con Wi-Fi pueden hacer decaer el interés entre los usuarios. Algunos especialistas apuntan que entre los posibles impactos negativos de la realidad aumentada sobre la experiencia turística se encuentran la confusión por exceso de información, la fatiga física o incomodidad de uso, la insatisfacción con la información recibida o la decepción con la calidad de los contenidos obtenidos y/o la forma de recibir la información (Yovcheva y otros, 2013).

Un tercer componente que puede mermar el uso de la realidad aumentada es el factor humano. Los usuarios no son máquinas y tienen sus limitaciones y preferencias a nivel cognitivo y de percepción. Los aspectos personales son clave y, seguramente, hacen que parte de los turistas prefiera seguir usando los medios convencionales de apoyo de la visita turística (guías en papel, mapas y planos, etc.). Será preciso, por tanto, personalizar y tener en cuenta las diferencias entre los usuarios (de género, de edad, de experiencia tecnológica, de origen y procedencia social, etc.) antes de proponer elementos de realidad aumentada para el consumo turístico. La habilidad para reconocer y para interpretar los estímulos visuales no es igual para todos, así como no todos tenemos la misma destreza para razonar a partir de estos estímulos de información recibidos. Será, por tanto, muy importante saber aportar la información precisa que requiere cada individuo, de manera clara y sin llegar a saturar ni a confundir al usuario.

\section{Conclusiones}

El repaso al potencial y a las limitaciones del uso de la realidad aumentada como herramienta para la mejora de la competitividad de los destinos turísticos nos lleva a cuatro conclusiones. Una primera afirmación es que parece claro que la realidad aumentada es una tecnología suficientemente interactiva y creativa y con grandes capacidades de transformar la experiencia turística del visitante cuando se aplica sobre los diferentes elementos del destino. El efecto sorpresa que puede generar y la vinculación directa con los usuarios explicarían la atracción que puede llegar a generar entre el público en general. Muy vinculado a este aspecto se deriva una segunda conclusión: se puede considerar que la realidad aumentada es una tecnología que puede liderar estrategias de innovación de los destinos basadas en las nuevas tecnologías. En tercer lugar, se puede concluir que la realidad aumentada como instrumento tecnológico al servicio de los consumidores turísticos aún tiene mucho camino por recorrer a la hora de mejorar los obstáculos que la limitan en cuanto a los aspectos tecnológicos, legales, de 
usabilidad y de personalización. Finalmente, se puede concluir también que la realidad aumentada puede jugar una baza importante en la definición de estrategias de competitividad para los destinos turísticos a partir del uso de las nuevas tecnologías siempre que los proyectos sean adecuadamente evaluados, tanto desde el punto de vista económico como social, y que se haga un balance adecuado de su coste-beneficio y de los impactos reales sobre el sector turístico y la satisfacción de los turistas y residentes.

\section{Bibliografía}

AGÜERO, A. M.; GONZÁLEZ, R. (2014). «Análisis de la aplicación de la realidad aumentada en el sector turístico: Una propuesta de mejora». Gran Tour: Revista de Investigaciones Turísticas. Núm. 10, pág. 52-72.

BUHALIS, D.; LAW, R. (2008). «Progress in information technology and tourism management: 20 years on and 10 years after the internet. The state of eTourism research». Tourism Management. Núm. 29, pág. 609-623.

BUHALIS, D.; O'CONNOR, P. «Information Communication Technology revolutionizing tourism». Tourism Recreation Research. Núm. 30 (3), pág. 7-16.

FRITZ, F.; SUSPERREGUI, A.; LINAZA, M. (2005). «Enhancing cultural tourism experiences with augmented reality technologies». 6th International Symposium on Virtual Reality, Archaeology and Cultural Heritage (VAST). Pisa.

GONZÁLEZ, F.; VILLAREJO, L.; MIRALBELL, O.; GOMIS, J. M (2012). «How to use mobile technology and augmented reality to enhance collaborative learning on cultural and natural heritage? An e-learning experience». New Trends on Global Education Conference 2012 (GEC 2012). North Cyprus, 24-26 de septiembre de 2012.

JEONG, M.; OH, H.; GREGOIRE, M. (2003). "Conceptualizing Web site quality and its consequences in the lodging industry». International Journal of Hospitality Management. Núm. 22 (2), pág. 161-175.

JUNG, T.; HAN, D. (2014). «Augmented Reality (AR) in Urban Heritage Tourism». e-Review of Tourism Research. Núm. 5.

KOUNAVIS, C.; KASIMATI, A. E.; ZAMANI, E. D. (2012). «Enhancing the tourism experience through mobile augmented reality. Challenges and prospects». International Journal of Engineering Business Management. Núm. 10 (4) [artículo en línea]. [Fecha de consulta: 2 de agosto de 2015]. http://cdn.intechopen.com/ pdfs/38051/InTech-Enhancing_the_tourism_experience_through_mobile_augmented_reality_challenges_ and_prospects.pdf

LAMFUS, C. y otros (2013). «Conceptualizing context in an intelligent mobile environment in travel and tourism». En: CANTONI, L.; XIANG, Z. (eds.). Information and communication technologies in tourism. Berlín: Springer, pág. $1-11$.

MAIN, H. (2001). "The expansion of technology in small and medium hospitality enterprises with a focus on net technology». Information Technology \& Tourism. Núm. 4 (3) (4), pág. 167-174.

YOVCHEVA, Z.; BUHALIS, D.; GATZIDIS, C. (2012). «Overview of smartphone augmented reality applications for tourism». e-Review of Tourism Research. Núm. 10 (2), pp. 63-66.

YOVCHEVA, Z.; BUHALIS, D.; GATZIDIS, C. (2013). «Engineering augmented tourism experiences». En: CANTONI, L.; XIANG, Z. (eds.). Information and communication technologies in tourism. Berlín: Springer, pág. 24-35. 


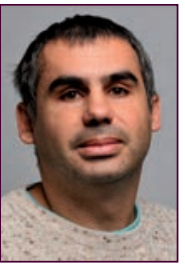

\section{Francesc González Reverté}

fgonzalezre@uoc.edu

Twitter @fgonzalezre

Profesor de la Universitat Oberta de Catalunya (UOC).

\section{Estudios de Economía y Empresa}

Doctor en Geografía por la UAB y profesor de los Estudios de Economía y Empresa de la UOC, en el grado de Turismo, desde el año 2003. Actualmente coordina el Grupo de Investigación Laboratorio del Nuevo Turismo de la UOC y es investigador del Grupo de Investigación de Análisis Territorial y Estudios Turísticos de la URV. Asimismo, es profesor del máster universitario de Turismo sostenible y TIC de la UOC. Sus principales preocupaciones y líneas de investigación se centran en el conocimiento del funcionamiento y el metabolismo de las ciudades turísticas de playa, la capacidad de los eventos turísticos para generar impactos sociales, las pautas de construcción de oferta de turismo responsable y la manera como la aplicación de las nuevas tecnologías puede mejorar la gestión de los destinos. Ha publicado diferentes artículos, libros y capítulos de libro sobre esta temática, entre los que destacan A propósito del turismo. La construcción social del espacio turístico, Planificación territorial del turismo o Ciudades efímeras. Transformando el turismo urbano a partir de la producción de eventos.

Los textos publicados en esta revista están -si no se indica lo contrario- bajo una licencia Reconocimiento-Sin obras derivadas 3.0 España de Creative Commons. Puede copiarlos, distribuirlos y comunicarlos públicamente siempre que cite su autor y la revista y la institución que los publica (autoría, nombre de la revista, institución editora); no haga con ellos obras derivadas. La licencia completa se puede consultar en http://creativecommons.org/licenses/by-nd/3.0/es/deed.es.

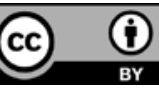

